\title{
Comparative Study between Planar and Bent Antenna Characterization
}

\author{
Lau Zheng Tung ${ }^{1}$, Ghafour Amouzed Mahdiraji ${ }^{1,}$ and Lee Chia Ping ${ }^{2}$ \\ ${ }^{1}$ School of Engineering, Taylor's University, 47500, Subang Jaya, Selangor, Malaysia \\ ${ }^{2}$ Heriot-Watt University Malaysia, 62200 Putra Jaya Malaysia
}

\begin{abstract}
Smaller designs of antenna usually implemented on Wi-Fi modules or navigation system devices which requires smaller bandwidth such as Planar Antenna. There are some antenna application requires bent antenna such as wearable UWB antenna but the substrate used will have some defects after bending and crumpling from time to time. What if the planar antenna is designed that it can be bent and the bending affects the impedance matching and shift in resonant frequency? So, this research aims to achieve the limitation of the planar antenna to be bent at certain angle and given frequency range $(1-8 \mathrm{GHz})$. The planar antenna is designed in the Computer Simulation Technology (CST) Software and the proposed antenna is then bend in the simulation software. The analysis of comparative evaluation for both planar and bent antenna is carried out on different characteristics such as evaluation between S-Parameters \& Frequency and evaluation between Voltage Standing Wavelength Ratio (VSWR) \& Frequency. The behaviour of radiation pattern for both antennas can also be obtained in the CST simulation.
\end{abstract}

\section{Introduction}

Antenna has been widely used in various form of communication in different applications from machine to machine. The application that requires the usage of antenna includes, radio broadcast, navigation systems, satellite communication, remote sensing in defence radar or medical scanning device as well. Planar antennas are commonly used in sensing device due to low cost and simple implementation with other system. The frequencies of the Planar antenna can reach as high as $77 \mathrm{GHz}$ which can be performed in automotive sensing devices. Typical antennas that people had worked on usually control by the different bandwidth and return loss of the signal during the receiving of the signal. While extensive of studies have been related to planar antenna, the studies on non-planar antenna such as bent antenna are limited. The bent antennas mostly are the solutions to some application as they are suitable to be implemented onto structures or objects that are not in planar form. There are researches identified the draw backs for bent antenna which would lead to certain distortion of parameters which includes, the impedance bandwidth,

${ }^{1}$ Corresponding author: Ghafour.AmouzadMahdiraji@,taylors.edu.my 
input impedance, directivity and phase angle when antenna is exposed to bending [1], [2]. Some cases where the substrate material is applied on the antenna with better parameters, allows the antenna to operate properly with better efficiency even though the antenna is bent [2].

$\mathrm{S}$ parameters are defined in terms of incident and reflected travelling waves which are simple to be used in analysis. S parameters are usually easy to be measured by using network analyzers and it is popular in any Radio Frequency (RF)/Microwave circuit design and testing. S Parameters are complex matrix that shows Reflection/Transmission characteristics (Amplitude/Phase) in frequency domain.

Figure 1 shows a two-port device which has four S-parameters. The first number following the "S" is port where the signal emerges while the second number is where the signal is applied. So, S21 is a measure of signal coming out from port 2 relative to RF stimulus entering port 1 . If the numbers are the same (S11), this indicates that the signal has a reflection measurement. Quantify of reflection and transmission characteristics of devices is available with amplitude and phase information. There are few measured terms are scalar in nature otherwise others are in vector. For example, return loss is a scalar measurement of reflection, while impedance results from a vector reflection measurement [3]. Meanwhile, like group delay, are referred to purely phase-related measurement. For Reflection, S11 /S22: the parameters that we can identify includes, Reflections (Return loss), Impedance, Admittance, VSWR. For Transmission, S21/S12: the parameters that we can identify includes, Gain/Loss (Insertion loss), Phase, Group delay (Delay time). To identify any loss of signals that was sent, a return signal of the transmission signal is needed which indicates the Reflection, S11 parameters. Return Loss is measured at the input to the coaxial cable connected to antenna and it is the difference between forward and reflected power in $\mathrm{dB}$. The Return Loss calculation is given by Radiated Power (PR) which the power is reflected back, divided by Incident Power (PT) which the power transmitted by the source. The Return Loss must be as small as possible for maximum power transfer and the ratio, PR/PT should also be as small as possible and the return loss should also have the value which is as large a negative as possible.

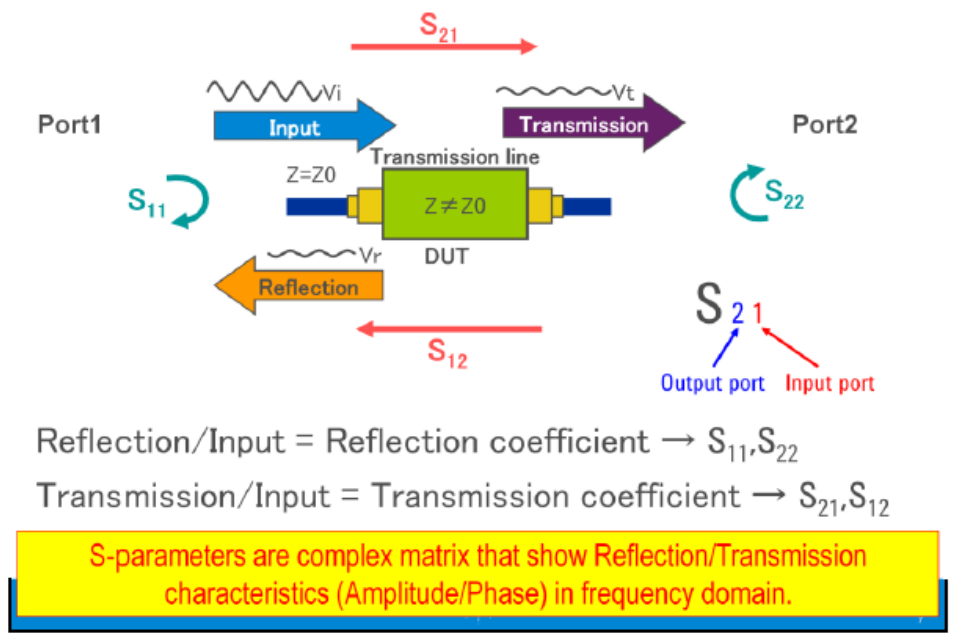

Fig. 1. Reflection/Transmission Characteristics (Amplitude/Phase) in Frequency domain [3].

\section{Methodology}




\subsection{Antenna design}

Figure 2 shows the antenna design with the dimensions. The antenna is designed on FR-4 substrate with the dimension given; dielectric constant, $\varepsilon_{r}$ of 4.3 , loss tangent of 0.025 and thickness of $0.16 \mathrm{~mm}$. The copper tape with thickness of $0.035 \mathrm{~mm}$ is used as conductive material for patch and ground. Most research recommended transmission line model to design the width, W and length, $L$ of the antenna [4], [5]. The implementation of transmission line model is fairly easy and antenna designs will acquire with reasonably good performance in terms of return loss and efficiency during the simulation of antenna. According to transmission line model, the length, $L$ and width, $\mathrm{W}$ of the patch are calculated as [5]:

\subsubsection{Calculation for patch width:}

The dimension of the antenna depends on the operating/resonant frequency and value of dielectric constant.

$$
W=\frac{c_{o}}{2 f_{o}} \sqrt{\frac{2}{\varepsilon_{r}+1}}
$$

Where $W$ is antenna width in mm, $c_{\mathrm{o}}$ is speed of light in free space with $3 \times 10^{8}, f_{\mathrm{o}}$ is operating frequency or resonant frequency, and $\varepsilon_{r}$ is dielectric constant of substrate.

\subsubsection{Calculation for effective permittivity}

The effective permittivity is important in the designing process of the antenna. There is radiation travelling through from patch towards the ground, followed by passing through air then passing through the substrate and this occurrence is referred as 'Fringing'. As we know that the air and substrate have different dielectric constant, so we need to find the effective of the dielectric constant [6].

$$
\varepsilon_{r e f f}=\frac{\left(\varepsilon_{r}+1\right)}{2}+\frac{\left(\varepsilon_{r}-1\right)}{2}\left(1+12 \frac{h}{W}\right)^{-0.5}
$$

Where $\varepsilon_{\text {eff }}$ is the effective permittivity, $h$ is the height of the substrate in mm, $\varepsilon_{r}$ is the dielectric constant of substrate and $W$ is the antenna with in $\mathrm{mm}$.

\subsubsection{Calculation for effective length:}

$$
L_{e f f}=\frac{c_{o}}{2 f_{o \sqrt{\varepsilon_{r e f f}}}}
$$

Where $L_{\text {eff }}$ is the effective length of patch, $\varepsilon_{\text {eff }}$ is the effective permittivity, $c_{\mathrm{o}}$ is speed of light in free space with $3 \times 10^{8}$, and $f_{\mathrm{o}}$ is operating frequency or resonant frequency. 


\subsubsection{Calculation for length extension:}

The size of the antenna is increased by an amount of length extension, $\Delta \mathrm{L}$ due to fringing [6].

$$
\Delta L=0.412 h\left[\frac{\left(\varepsilon_{\text {reff }}+0.3\right)\left(\frac{W}{h}+0.264\right)}{\left(\varepsilon_{\text {reff }}-0.258\right)\left(\frac{W}{h}+0.8\right)}\right]
$$

Where $\Delta L$ is the length extension, $\varepsilon_{\text {eff }}$ is the effective permittivity, $h$ is the height of the substrate in $\mathrm{mm}$, and $W$ is the antenna width in $\mathrm{mm}$.

\subsubsection{Calculation for patch length:}

$$
L=L_{e f f}-2 \Delta L
$$

Where $L$ is the length of patch in mm, $L_{\text {eff }}$ is the effective length of patch and $\Delta L$ is the length extension. 

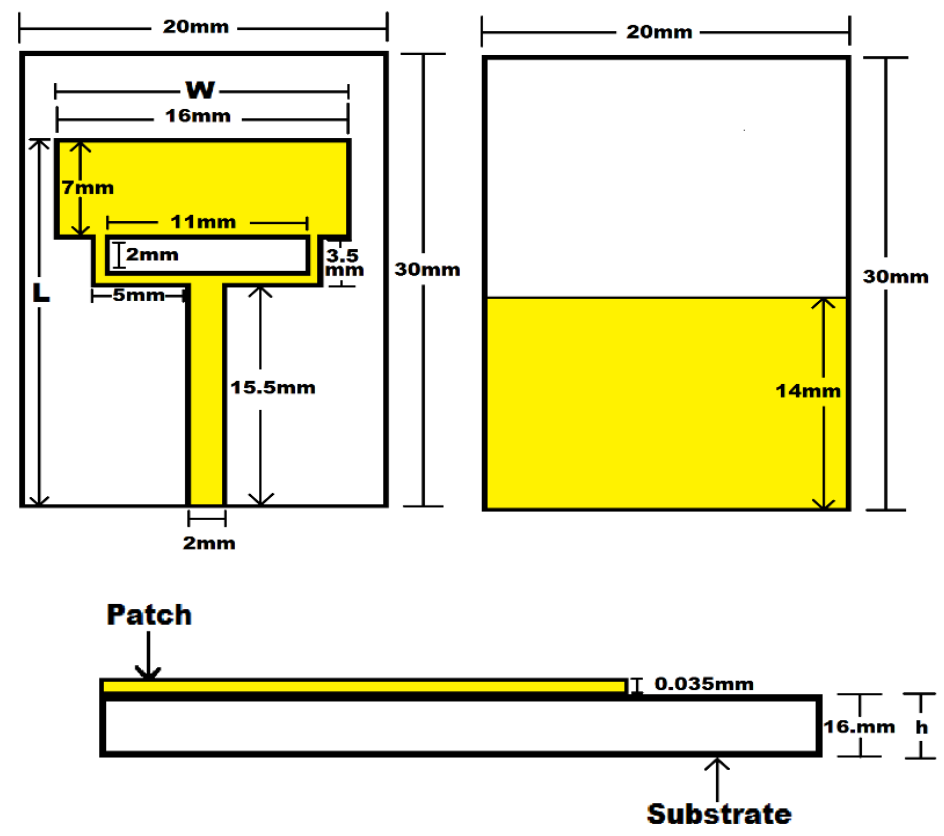

Fig. 2. Parameter Design of Proposed Antenna.

\subsection{Antenna bending}

To demonstrate the bending of the antenna, Figure 3 shows a cylinder with radii of 5-10 $\mathrm{mm}$. The bending function is visualized to bend on a cylindrical surface in CST software. Figure 4 shows the bending direction towards the cylindrical surface. The antenna is bend on XZ plane which bends from both sides of the antenna. The antenna is tested on different type of radii bending of the cylinder. While the bending of antenna is increased, the radii of the cylinder is decreased. The results of S parameters, VSWR and Radiation Pattern is

simulated. 


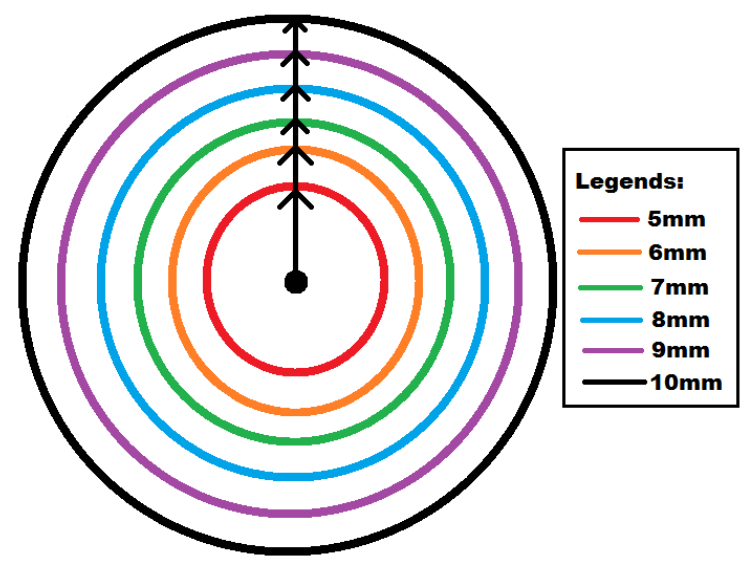

Fig. 3. Different radii of the cylinder.

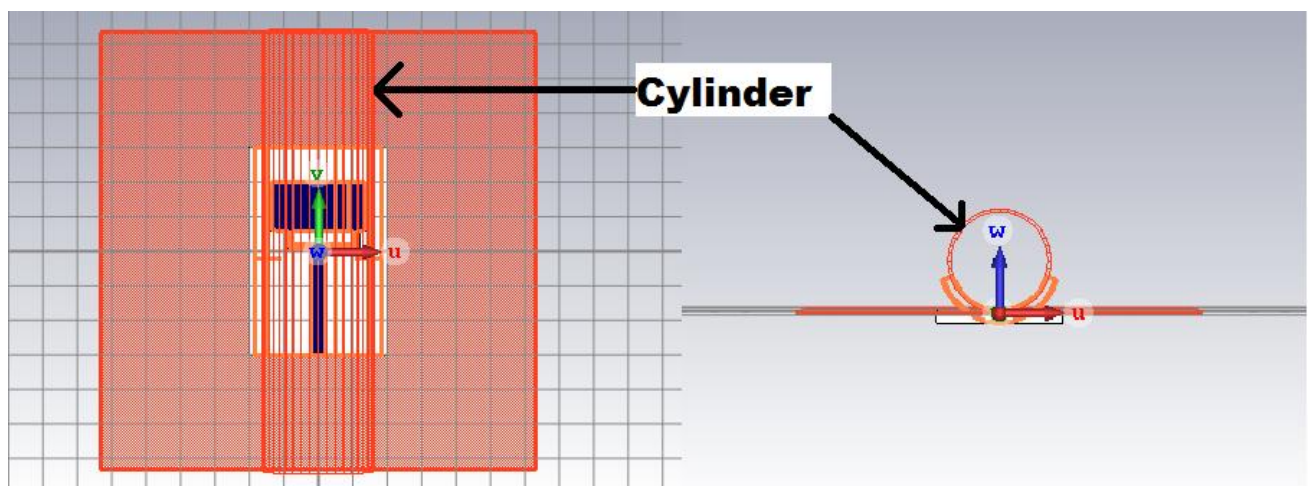

Fig. 4. Cylinder object for the antenna to bent towards in CST.

\section{Result and discussion}

\subsection{Planar antenna performance}

Simulations are carried out using Computer Simulation Software (CST) to simulate the behaviour of the antenna. Figures 5 and 6 show the simulated results in terms of return loss and VSWR of the antenna. The simulated return loss shows an impedance bandwidth of $2.67 \mathrm{GHz}(3.08 \mathrm{GHz}-5.75 \mathrm{GHz})$ which is in a good operate operation. The VSWR shows a huge drop for $1 \mathrm{GHz}$ to $3 \mathrm{GHz}$. One of the reason is that the frequency range is not suitable to simulate a better VSWR result since the VSWR is more than 2 . The results starting from $3 \mathrm{GHz}$ to $8 \mathrm{GHz}$ has a better measurement in the VSWR which satisfies the VSWR ratio requirement of less than 2 . In order to test and determine the performance of the planar antenna, the planar antenna is then bent in the $\mathrm{XZ}$ plane with different cylinder radii $(\mathrm{r}=5,6,7,8,9$ and $10 \mathrm{~mm})$. The results of the bent design are compare with the results of planar design. 


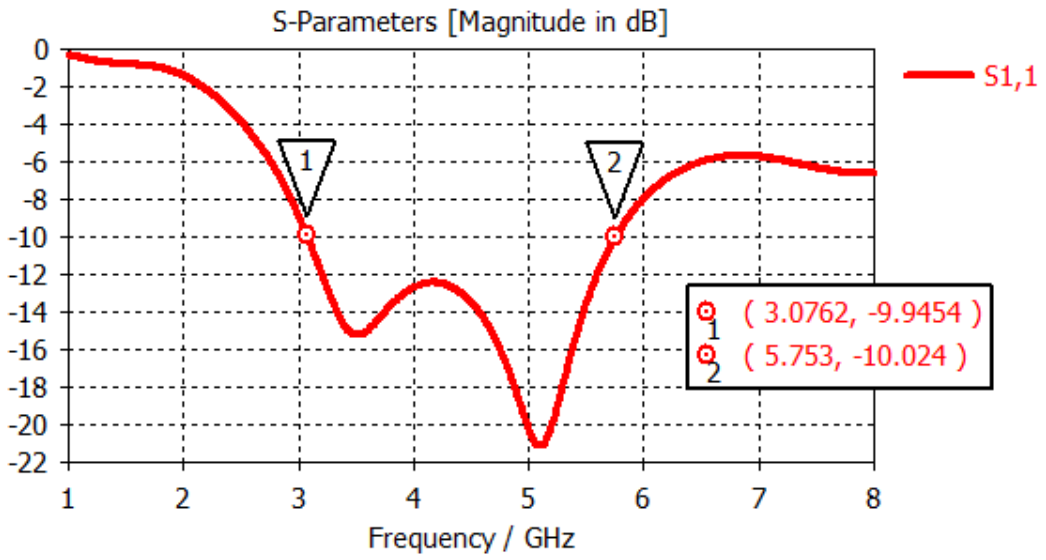

Fig. 5. Simulated S Parameter for Planar Antenna.

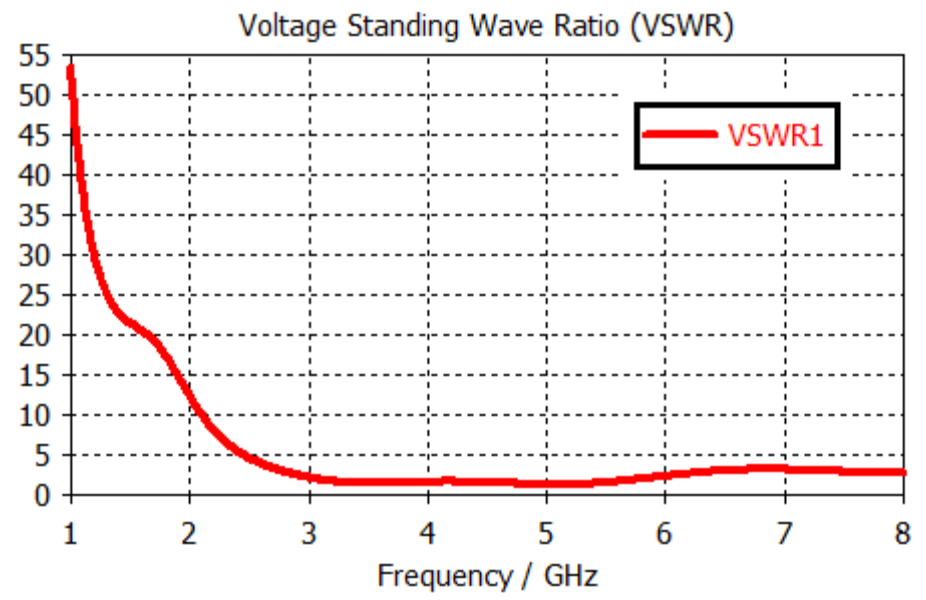

Fig. 6. Simulated VSWR for Planar Antenna.

\subsection{Effects on return loss and bandwidth}

Figure 7 compares planar antenna and bent antenna that is cylindrical bent with different radii of 5-10 $\mathrm{mm}$. Theoretically, the antenna changes its effective length when the antenna is bent. The bending on the antenna increases, the effective length is decreases along and the resonant frequency will shift to a higher band [7]. Table 1 show that the bandwidth is large (in GHz) due to the low permittivity of the dielectric substrate [2]. This explains that lower permittivity dielectric substrate with thicker in thickness is able to produce an antenna design with high efficiency and large bandwidth. Meanwhile, dielectric substrate with high permittivity will result in a lower efficiency in the antenna design results. The return loss shows increment along as the bending increases which means the antenna is operating normally. Generally, a larger return loss results in more energy is delivered into the antenna. The antenna is to be design in wide frequency range and the wider of the bandwidth, the better in the antenna performance. The antenna operates normally within the desire range even though there is a shift in frequency. The performance of the proposed 
antenna is slightly decreased while the antenna bending is increased but the antenna still operates efficiently at resonant frequency of $4.591 \mathrm{GHz}$.

S-Parameters [Magnitude in $\mathrm{dB}$ ]

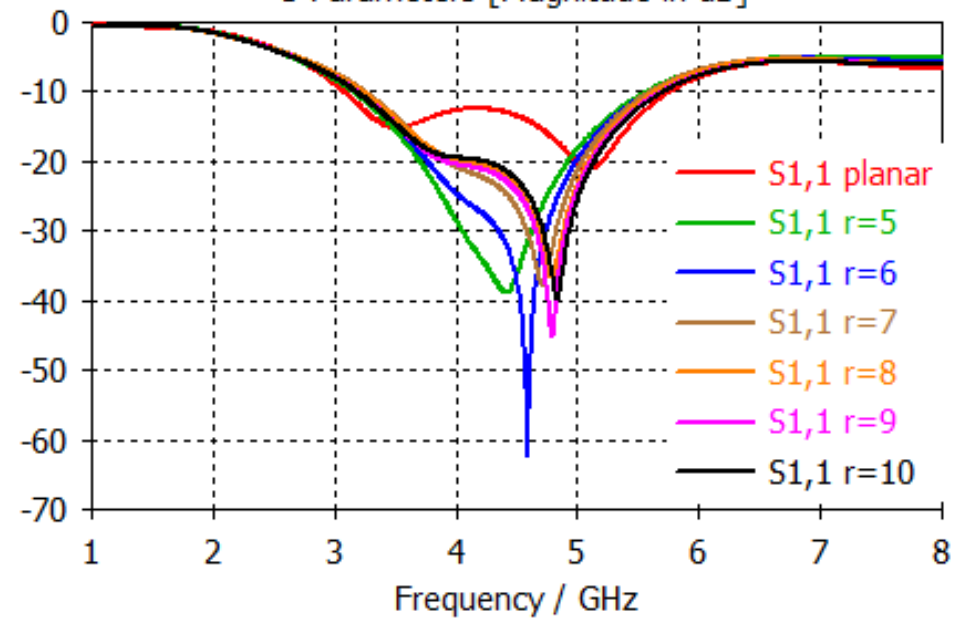

Fig. 3. Variation of return loss with bending.

Table 1. Change in radiation characteristics of antenna with bending.

\begin{tabular}{|c|c|c|}
\hline $\begin{array}{c}\text { Cylindrical Bend } \\
{[\mathrm{mm}]}\end{array}$ & $\begin{array}{c}\text { Return Loss at 4.591 } \\
\text { GHz [dB] }\end{array}$ & Bandwidth [GHz] \\
\hline $\mathrm{r}=0$ (planar) & -14.17 & $2.68(5.76-3.08)$ \\
\hline $\mathrm{r}=5$ & -31.56 & $2.40(5.56-3.16)$ \\
\hline $\mathrm{r}=6$ & -62.32 & $2.40(5.59-3.19)$ \\
\hline $\mathrm{r}=7$ & -30.14 & $2.35(5.59-3.24)$ \\
\hline $\mathrm{r}=8$ & -26.12 & $2.40(5.63-3.23)$ \\
\hline $\mathrm{r}=9$ & -26.78 & $2.50(5.68-2.50)$ \\
\hline $\mathrm{r}=10$ & -24.63 & $2.52(5.70-3.18)$ \\
\hline
\end{tabular}




\subsection{Effect on voltage standing wave ratio (VSWR)}

Figure 8 shows the graph of VSWR comparison between both planar antenna and bent antennas. The graph shows that the bent antennas has a similar trend as the planar antenna in terms of VSWR. From the generated Voltage Standing Wave Ratio (VSWR), the results are taken from the resonant frequency at $4.591 \mathrm{GHz}$. Table 2 shows the percentage of power delivered by the receiver reflected from the antenna. The maximum power reflected is $3.8 \%$ which $96.2 \%$ of the power is delivered to the antenna. The minimum power reflected is $5.86 \mathrm{E}-05 \%$ which $99.99 \%$ of the power is delivered to the antenna. The antennas performance is considered good and little would be gained by the impedance matching since the simulated VSWR results are less than 2. The VSWR from $1 \mathrm{GHz}$ to 3 $\mathrm{GHz}$ shows a high VSWR which means that the range of frequency is reflecting a higher power back to the antenna which may affect the antenna performance. After that, the VSWR is within the acceptable range which most of the antenna VSWR are below 2.

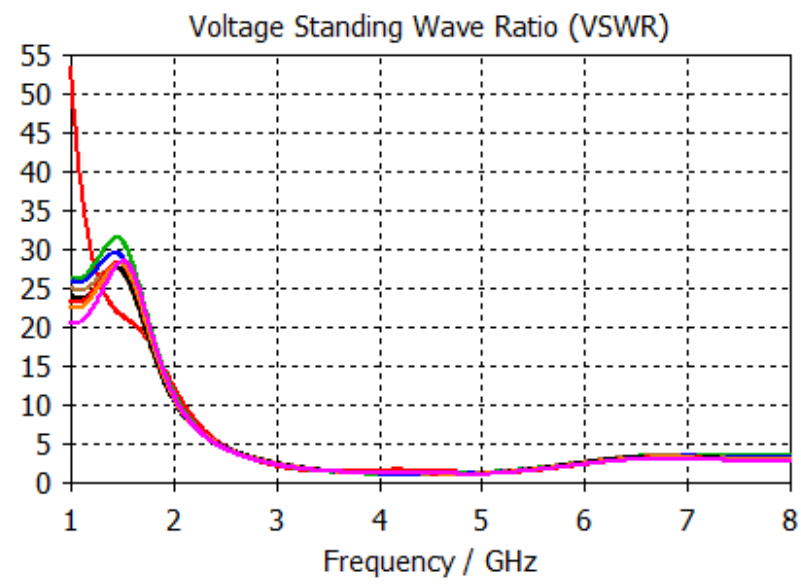

Fig. 8. Comparison of planar antenna and bent antenna in terms of Voltage Standing Wave Ratio.

Table 2. Change in VSWR of antenna with bending.

\begin{tabular}{|c|c|c|c|c|}
\hline $\begin{array}{c}\text { Cylindrical Bend, } \\
r \\
{[\mathrm{~mm}]}\end{array}$ & VSWR & $\begin{array}{c}\text { Return Loss at } \\
4.591 \\
\mathrm{GHz}[\mathrm{dB}]\end{array}$ & $\begin{array}{c}\text { Reflection Coefficient, } \\
\Gamma\end{array}$ & $\begin{array}{c}\text { Reflected Power } \\
(\%)\end{array}$ \\
\hline 0 & 1.49 & -14.17 & 0.19 & 3.82 \\
\hline 5 & 1.05 & -31.56 & 0.03 & 0.069 \\
\hline 6 & 1.00 & -62.32 & 0.0007 & $5.86071 \mathrm{E}-05$ \\
\hline 7 & 1.06 & -30.14 & 0.031 & 0.10 \\
\hline 8 & 1.10 & -26.12 & 0.049 & 0.24 \\
\hline 9 & 1.10 & -26.78 & 0.046 & 0.21 \\
\hline 10 & 1.12 & -24.63 & 0.059 & 0.34 \\
\hline
\end{tabular}




\subsection{Effect on radiation pattern}

Figure 9 shows the 2D radiation patterns for planar and bent antenna on XZ plane. Table 3 shows the angular width $(3 \mathrm{~dB})$ tends to decrease along with the increase in bending of the antenna. This leads to shrink in the radiation pattern in bending plane which the gain and efficiency of the antenna increases with the bending. The antenna gain, $G$ is related to directivity, D and antenna efficiency of the antenna which the efficiency of the antenna increases along with the bending of antenna.

\section{Farfield Directivity Abs (Phi=90)}

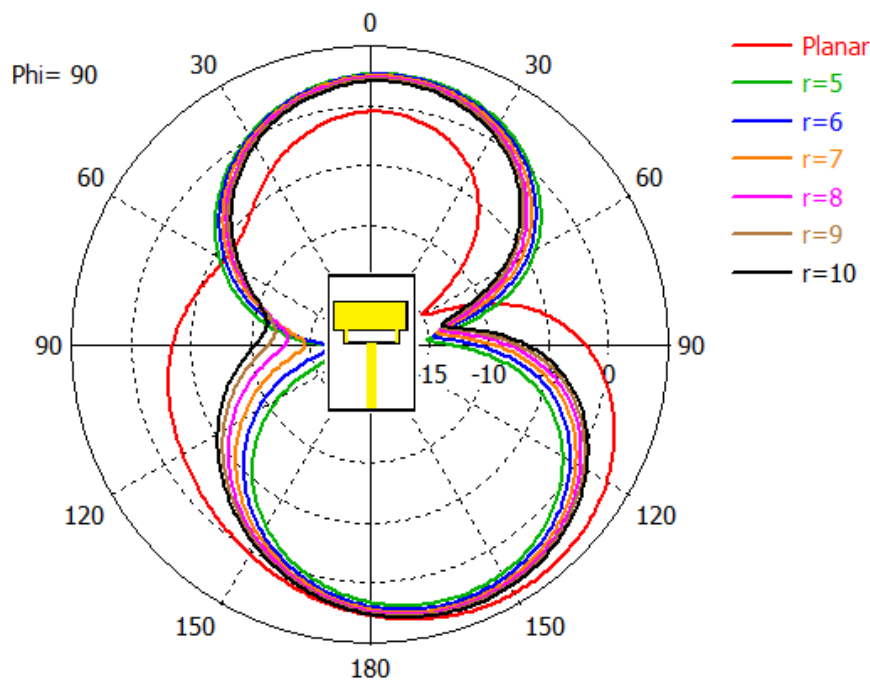

Theta / Degree vs. dBi

Fig. 4. Radiation pattern of planar antenna and bent antenna.

Table 3. Change in radiation pattern characteristics of antenna with bending.

\begin{tabular}{|c|c|c|c|c|c|c|}
\hline $\begin{array}{c}\text { Antenna } \\
\text { Radii, } r \\
{[\mathrm{~mm}]}\end{array}$ & $\begin{array}{c}\text { Main Lobe } \\
\text { Magnitude } \\
{[\mathrm{dBi}]}\end{array}$ & $\begin{array}{c}\text { Main Lobe } \\
\text { Direction } \\
{[\mathrm{deg} .]}\end{array}$ & $\begin{array}{c}\text { Angular } \\
\text { width }(3 \mathrm{~dB}) \\
{[\mathrm{deg} .]}\end{array}$ & $\begin{array}{c}\text { Gain } \\
(\mathrm{dB})\end{array}$ & $\begin{array}{c}\text { Directivity } \\
(\mathrm{dBi})\end{array}$ & $\begin{array}{c}\text { Efficiency } \\
(\%)\end{array}$ \\
\hline 0 (Planar) & 3.93 & 147.0 & 96.0 & 1.00 & 3.93 & 25.5 \\
\hline 5 & 2.70 & 6.0 & 78.0 & 1.33 & 2.70 & 49.5 \\
\hline 6 & 2.61 & 5.0 & 75.7 & 1.06 & 2.61 & 40.6 \\
\hline 7 & 2.86 & 159.0 & 80.9 & 0.89 & 2.86 & 31.2 \\
\hline 8 & 3.07 & 158.0 & 82.7 & 0.75 & 3.06 & 24.5 \\
\hline 9 & 3.22 & 158.0 & 84.1 & 0.79 & 3.21 & 24.7 \\
\hline 10 & 3.33 & 157.0 & 85.3 & 0.83 & 3.33 & 24.9 \\
\hline
\end{tabular}

\section{Conclusion and future work}

The paper is focused on the limitation of planar antenna performances when the antenna design is bent at certain degrees at given frequency range $(1-8 \mathrm{GHz})$. The design of planar antenna is executed in the Computer Simulation Technology (CST) Software. In this research, the result shows that the performance of the proposed antenna is slightly decreased while the antenna bending is increased but the antenna still operates efficiently 
on the resonant frequency of $4.591 \mathrm{GHz}$. The results of VSWR between both designs are within the acceptable range after $3 \mathrm{GHz}$ onwards. The angular width decreases in the plane of bending which results in increase in the antenna gain. The antenna still operates efficiently from $25.5 \%$ to $49.5 \%$ with bending on the resonant frequency at $4.591 \mathrm{GHz}$ even after bending. Based on the obtained conclusion, the antenna design can be implemented as a Wearable Antenna application. In future work, the performance analysis of the antenna in different angle will be done as well as trying on the different type of material used for the antenna substrate.

\section{Reference}

[1] N. A. ELIAS, N. A. Samsuri, M. K. A. Rahim, C. J. Panagamuwa, and W. G. Whittow, J. Teknol., 77 (2015)

[2] S. Dey, N. Saha, and A. Alomainy, Antennas and Propagation Conference (LAPC) (2011)

[3] "S-parameter measurement Basics for High-Speed Digital Engineers." [Online]. Available: http://literature.cdn.keysight.com/litweb/pdf/5991-3736EN.pdf. [Accessed: 20-Oct-2017].

[4] Y. Ahmed, Y. Hao, and C. Parini, Int. J. Antennas Propag., 2008 (2008).

[5] V. Bernard, J. Paul, and I. Iloh, Microstrip Antenna Des. Using Transm. Line Model, 3 (2013).

[6] M. A. Afridi, Biol. Chem. Res., 2015 (2015).

[7] M. Rizwan, L. Sydänheimo, and L. Ukkonen, Prog. Electromagn. Res. Symp., 2015January (2015). 